\title{
TOPOLOGICAL ENTROPY ON SADDLE SETS IN $\mathbf{P}^{2}$
}

\author{
JEFFREY DILLER AND MATTIAS JONSSON
}

\begin{abstract}
We consider hyperbolic sets of saddle type for holomorphic mappings in $\mathbf{P}^{2}$. Our main result relates topological entropy on such sets to a normal families condition on local unstable manifolds.
\end{abstract}

\section{INTRODUCTION}

The Fatou set $\mathcal{F}$ of a holomorphic map $f: \mathbf{P}^{k} \circlearrowleft$ is the largest open subset of $\mathbf{P}^{k}$ on which iterates of $f$ form a normal family. The complement of $\mathcal{F}$ is called the Julia set when $k=1$, and it is well-known that the Julia set is the closure of the set of repelling periodic points. When $k=2$, however, even product maps suffice to show that the structure of $\mathbf{P}^{2} \backslash \mathcal{F}$ is more intricate. For instance, $\mathbf{P}^{2} \backslash \mathcal{F}$ contains both repelling periodic points and periodic points of "saddle" type - with one expanding and one contracting direction. In nice situations, these distinct types of periodic points occupy distinct regions in $\mathbf{P}^{2} \backslash \mathcal{F}$, and each of these smaller regions legitimately vies with $\mathbf{P}^{2} \backslash \mathcal{F}$ for the designation of Julia set.

Our concern in this paper is with what we call saddle sets of a holomorphic map $f: \mathbf{P}^{2} \circlearrowleft$. These generalize the notion of a saddle periodic point, and while we defer the precise definition until Section 1, the following description will suffice for the moment. A closed invariant set $\Lambda=f(\Lambda) \subset \mathbf{P}^{2}$ is a saddle set of $f$ if $f$ acts transitively and hyperbolically on $\Lambda$ with one contracting and one expanding direction, and if $\Lambda$ is in some sense both maximal and isolated as a hyperbolic set. Important examples of saddle sets are given by the basic sets of saddle type for an Axiom A map $f: \mathbf{P}^{2} \circlearrowleft$. Indeed the paper [FS2] of Fornæss and Sibony on Axiom A holomorphic maps of $\mathbf{P}^{2}$ inspired much of the work on which this paper is based.

Given a history $\hat{p}=\left(p_{j}\right)_{j \leq 0}$ in $\Lambda$ (i.e. $p_{j} \in \Lambda$ and $f\left(p_{j-1}\right)=p_{j}$ for all $j \leq 0$ ) and a small fixed $\delta>0$, the associated local unstable manifold is

$$
W_{\text {loc }}^{u}(\hat{p})=\left\{q \in \mathbf{P}^{2}: \operatorname{dist}\left(q_{j}, p_{j}\right)<\delta \text { for } j \leq 0 \text { and some } \hat{q} \text { with } q_{0}=q\right\} .
$$

As is well-known, $f$ expands local unstable manifolds near $\Lambda$. Nevertheless, we call $\Lambda$ terminal if for each history $\hat{p}$ in $\Lambda$, iterates of $f$ act normally on $W_{\text {loc }}^{u}(\hat{p})-\Lambda$. We believe that terminal saddle sets play a distinguished role in the global dynamics of $f$. Our main result is

Theorem A. Let $\Lambda$ be a saddle set of a holomorphic map $f: \mathbf{P}^{2} \circlearrowleft$ of degree $d$. Then the topological entropy $h_{\mathrm{top}}\left(\left.f\right|_{\Lambda}\right)$ of $f$ restricted to $\Lambda$ is no greater than $\log d$. Equality holds if and only if $\Lambda$ is terminal.

Date: May 16, 2005.

1991 Mathematics Subject Classification. Primary: 32F50, Secondary: 58F15, 58F23.

Key words and phrases. entropy, holomorphic dynamics, hyperbolicity, saddle sets .

First author supported by NSF grant no DMS98-96370. Second author supported by STINT. 
Topological entropy is, roughly speaking, a non-negative number that measures the complexity of orbits of $f$ contained in $\Lambda$. We do not actually use the precise definition of topological entropy in this paper, so we refer the reader to the book $[\mathrm{KH}]$ for such a definition, but we do point out that for any saddle set $\Lambda$,

$$
h_{\mathrm{top}}\left(\left.f\right|_{\Lambda}\right)=\lim _{n \rightarrow \infty} \frac{1}{n} \log \#\left\{p=f^{n}(p) \in \Lambda\right\} .
$$

So if, as in the Axiom A case, all saddle periodic points lie in saddle sets of $f$, then either the vast asymptotic majority of these points will lie in terminal saddle sets, or (in the absence of terminal saddle sets) saddle periodic points will be comparatively scarce.

Notice that Theorem A connects the behavior of $f$ on $\Lambda$ to the global behavior of $f$ on $\mathbf{P}^{2}$. That is, topological entropy is a quantity purely intrinsic to the action of $f$ on $\Lambda$. On the other hand, large iterates of $f$ might a priori take the local unstable manifolds of $\Lambda$ anywhere in $\mathbf{P}^{2}$. Thus the normal families criterion underlying terminality is really a global condition on the behavior of $f$.

Despite the topological nature of the hypothesis and conclusion of Theorem A, the proof actually relies largely on measure theory. This dependence happens in two ways. First of all, there is a natural positive closed $(1,1)$ current $T$ associated with any holomorphic map of $\mathbf{P}^{2}$ with degree greater than one. This current is globally defined and has the transformation property $f^{*} T=d \cdot T$. It also has the property that $\operatorname{supp} T$ is the complement of the Fatou set. Secondly, as Ruelle and Sullivan $[\mathrm{RS}]$ observed in the case of Axiom A diffeomorphisms, there is a canonical local current $\sigma^{u}$ supported on the local unstable manifolds of a saddle set $\Lambda$. This local current satisfies $f_{*} \sigma^{u}=\lambda \cdot \sigma^{u}$ where $\lambda=h_{\text {top }}\left(\left.f\right|_{\Lambda}\right)$. It is possible to understand the wedge product $\sigma^{u} \wedge T$ as a positive measure defined near $\Lambda$, and the proof of Theorem A proceeds by considering the support and invariance of this measure.

Bedford and Smillie [BS] were the first to realize the importance of currents like $\sigma^{u}$ for multivariable complex dynamics. At least in the terminal case, the current $\sigma^{u}$ that we use here is identical with the current $\sigma$ constructed by very different means in [FS2, Theorem 5.10]. We plan to explore this and other properties of $\sigma^{u}$ further in a future paper.

It is not difficult to give examples of terminal saddle sets. We present several in Section 3. It is harder to find examples of non-terminal saddle sets. Nevertheless, we show

Theorem B. There exist holomorphic maps of $\mathbf{P}^{2}$ with non-terminal saddle sets. More precisely, given integers $d \geq 2$ and $0<k<d$ one can find a holomorphic map of degree $d$ with a saddle set $\Lambda$ such that $h_{\mathrm{top}}\left(\left.f\right|_{\Lambda}\right)=\log k$.

The proof of this theorem is constructive. That is, we actually manufacture examples of the desired type. These examples all come from the family of skew product maps, which have been studied in their own right by the second author [J2, J3] and Heinemann [H1, H2]. The fact that our examples fail to be terminal follows from Theorem A.

The contents of the rest of this paper are arranged as follows. Section 1 contains most of the background needed for this paper, including a review of the hyperbolic theory, the constructions of $\sigma^{u}$ and $T$, and the (precise) definition of a saddle set. Section 2 provides the proof of Theorem A. Section 3 presents examples of 
both terminal and non-terminal saddle sets. In particular, it contains the proof of Theorem B.

\section{BACKGROUND}

In this section we review the theory of hyperbolic dynamical systems. In particular, we describe the transversal measure and laminar current associated to the local unstable manifolds of a hyperbolic set. Our presentation is biased in two important ways. First of all, we are interested in non-invertible maps, and this leads to extra subtlety in the definition and properties of a hyperbolic set. The first paper in the second author's thesis [J1] provides further detail about hyperbolicity in the non-invertible setting. Secondly, we seek to prove results that are largely semi-local in nature. That is, we will usually be concerned with assertions that are valid only in a small neighborhood of a hyperbolic set. After generalities about hyperbolicity, we will discuss the hyperbolic sets of particular interest to us and indicate how such sets arise naturally for maps satisfying the (global) Axiom A condition. We will close the section with a brief review of the definition and properties of the global current $T$ associated with a holomorphic map $f: \mathbf{P}^{2} \circlearrowleft$.

1.1. Hyperbolicity. Let $f: X \circlearrowleft$ be a holomorphic (possibly branched), finiteto-one map of a compact complex manifold $X$. Suppose that $\Lambda=f(\Lambda) \subset X$ is a compact subset. We define the natural extension $\hat{f}: \hat{\Lambda} \circlearrowleft$ to be the induced map on histories contained in $\Lambda$. That is, a point in $\hat{\Lambda}$ is a sequence $\hat{p}=\left(p_{j}\right)_{j \leq 0}$ of points $p_{j} \in \Lambda$ such that $f\left(p_{j}\right)=p_{j+1}$, and $\hat{f}$ is the shift map sending $\left(p_{j}\right)$ to $\left(p_{j+1}\right)$. We give $\hat{\Lambda}$ the product topology induced from $X^{\infty}$ so that $\hat{f}$ is a homeomorphism of a compact metric space. For every $j \leq 0$ we define the projections $\pi_{j}: \hat{\Lambda} \rightarrow \Lambda$ by $\pi_{j}(\hat{p})=p_{j}$. We obtain a vector bundle $\widehat{T \Lambda}$ over $\hat{\Lambda}$ by using $\pi_{0}$ to pull back the tangent bundle of $X$. Points in this bundle are specified by $(\hat{p}, \mathbf{v})$ where $\mathbf{v}$ is tangent to $X$ at $p_{0}$.

We will say that $f$ is hyperbolic on $\Lambda$ if there is a continuous $\hat{f}$-invariant splitting $\widehat{T \Lambda}=E^{s} \oplus E^{u}$ and constants $C>0, \rho>1$ such that

$$
\left\|D f^{n} \mathbf{v}\right\| \leq C \rho^{-n}\|\mathbf{v}\|, \quad\left\|\left(D f^{n}\right)^{-1} \mathbf{w}\right\| \leq C \rho^{-n}\|\mathbf{w}\|
$$

for $n \geq 0$ and all $\mathbf{v} \in E^{s}$ and $\mathbf{w} \in E^{u}$. The inequalities imply the choice of an Hermitian metric on $X$, but any such metric will do. After a continuous change of metric near $\Lambda$, one can assume (as we will do) that the constant $C$ is 1 . Note that the definition of hyperbolicity requires only that $D f$ be invertible on the space $E^{u}$.

While $E^{u}(\hat{p})$ necessarily depends on the entire history $\hat{p}$ of the point $p=p_{0}$, characterization in terms of forward iteration implies that $E^{s}(\hat{p})=E^{s}(p)$ depends only on $p$ and can therefore be considered a subspace of $T X_{p}$. In particular, the dimensions of $E^{s}$ and $E^{u}$ are constant on $\Lambda$ if we assume that $\left.f\right|_{\Lambda}$ is topologically transitive - an assumption we now adopt for the sake of simplicity. As advertised in the introduction, our concern in this paper is with hyperbolic sets in $\mathbf{P}^{2}$ where both $E^{u}$ and $E^{s}$ have dimension one.

The primary consequence of hyperbolicity is the existence of local stable and unstable manifolds attached to points in the hyperbolic set (see for example, [Sh] 
or [PS]). For small enough $\delta>0$ and for any history $\hat{p} \in \hat{\Lambda}$, the sets

$$
\begin{aligned}
W_{\delta}^{s}(p) & =\left\{q \in X: \operatorname{dist}\left(f^{n}(q), f^{n}\left(p_{0}\right)\right)<\delta \text { for all } n \geq 0\right\} \\
W_{\delta}^{u}(\hat{p})=\{q \in X: \text { there exists a history } \hat{q} \in \hat{X} \text { of } q & \text { such that } \left.\operatorname{dist}\left(q_{n}, p_{n}\right)<\delta \text { for all } n \leq 0\right\}
\end{aligned}
$$

are $f$-invariant complex submanifolds tangent to $E^{s}(p)$ and $E^{u}(\hat{p})$, respectively. These are the local stable and unstable manifolds of $p$ and $\hat{p}$, respectively. As the notation implies, there is a unique local stable manifold but possibly many local unstable manifolds passing through a point $p \in \Lambda$. Where it is not important to be explicit about $\delta$, we shall write $W_{\text {loc }}^{s}(p)$ and $W_{\text {loc }}^{u}(\hat{p})$.

If we restrict to $\Lambda$, then we can lift the local stable and unstable manifolds to the natural extension $\hat{\Lambda}$, provided that we are willing to settle for a bit more asymmetry in the results. We define $\hat{W}_{\text {loc }}^{s}(p)=\pi_{0}^{-1}\left(W_{\text {loc }}^{s}(p) \cap \Lambda\right)$ simply by pulling back. On the other hand we let $\hat{W}_{\text {loc }}^{u}(\hat{p})=\left\{\hat{q} \in \hat{\Lambda}: q_{j} \in W_{\text {loc }}^{u}\left(\hat{f}^{j}(\hat{p})\right)\right.$ for all $\left.j \leq 0\right\}$ consist of only those histories $\hat{q}$ of points $q \in W_{\text {loc }}^{u}(\hat{p})$ that are backward asymptotic to $\hat{p}$. The projection $\pi_{0}$ is not generally injective on $\hat{W}_{\text {loc }}^{s}(p)$, but since $f$ acts injectively on local unstable manifolds, $\pi_{0}: \hat{W}_{\text {loc }}^{u}(\hat{p}) \rightarrow W_{\text {loc }}^{u}(\hat{p})$ is a homeomorphism onto its image. We observe that it is possible to take a more intrinsic approach to defining local stable/unstable sets in $\hat{\Lambda}$, but for our purposes, it is more convenient to use the above definitions.

Continuous variation of the splitting and compactness of $\Lambda$ guarantee that a local stable manifold $W_{\text {loc }}^{s}(p)$ intersects a local unstable manifold $W_{\text {loc }}^{u}(\hat{q})$ in at most one point. It also guarantees that the intersection will be non-empty if $q_{0}$ and $p_{0}$ are close enough. It turns out to be quite useful to know that the intersection lies in $\Lambda$ and, stronger still, that the intersection has a unique history lying in $\hat{\Lambda}$. In other words,

Definition 1.1. We say that $\hat{\Lambda}$ has local product structure if there exist constants $\delta, \delta^{\prime}>0$ such that for all $p \in \Lambda$ and all $\hat{q} \in \hat{\Lambda}$ such that $\operatorname{dist}\left(p, q_{0}\right)<\delta^{\prime}$, the intersection $\{[p, \hat{q}]\} \stackrel{\text { def }}{=} \hat{W}_{\delta}^{s}(p) \cap \hat{W}_{\delta}^{u}(\hat{q})$ consists of a unique point in $\hat{\Lambda}$.

Local product structure is equivalent (see [J1]) to the following local maximality condition: there exists a neighborhood $\mathcal{N}$ of $\Lambda$ such that any full orbit in $\mathcal{N}$ is actually contained in $\Lambda$. We remark that $[\cdot, \cdot]$ commutes with $f$. That is, $\hat{f}([p, \hat{q}])=$ $[f(p), \hat{f}(\hat{q})]$. If $\hat{\Lambda}$ has local product structure, then continuous variation of local stable/unstable manifolds implies that the map $[\cdot, \cdot]: W_{\text {loc }}^{u}(\hat{p}) \times \hat{W}_{\text {loc }}^{s}(p) \rightarrow \hat{\Lambda}$ is a homeomorphism onto a neighborhood (in $\hat{\Lambda})$ of $\hat{p}$. In particular, $\pi_{0}: \hat{W}_{\text {loc }}^{u}(\hat{p}) \rightarrow$ $W_{\text {loc }}^{u}(\hat{p}) \cap \Lambda$ is surjective.

1.2. Transversal measures and laminar currents. We continue our discussion with the additional assumption in this subsection that $f$ is topologically mixing on $\Lambda$. Local product structure on $\hat{\Lambda}$ allows us to define holonomy along unstable manifolds. Given $p \in \Lambda$, we define the unstable holonomy map $\hat{\chi}_{p}^{u}: R \rightarrow \hat{W}_{\text {loc }}^{s}(p)$ on a neighborhood $R \subset \hat{\Lambda}$ of $\hat{W}_{\text {loc }}^{s}(p)$ by $\hat{\chi}_{p}^{u}(\hat{q})=[p, \hat{q}]$. The restriction $\hat{\chi}_{q, p}^{u}$ of $\hat{\chi}_{p}^{u}$ to $R \cap \hat{W}_{\mathrm{loc}}^{s}(q)$ is a homeomorphism onto its image.

Ruelle-Sullivan $[\mathrm{RS}]$ and Bowen-Marcus [BM] considered the notion of transversal measures for Axiom A diffeomorphisms. A translation of their results into our setting reads as follows. 
Theorem 1.2. Given $f, \Lambda$ as above, there exists for each $p \in \Lambda$ a positive measure $\hat{\mu}_{p}^{u}$ on $\hat{W}_{\mathrm{loc}}^{s}(p)$ with the following properties:

(1) If $\hat{\chi}_{p, q}^{u}(E)=F$ for Borel sets $E \subset \hat{W}_{\text {loc }}^{s}(q)$ and $F \subset \hat{W}_{\text {loc }}^{s}(p)$, then $\hat{\mu}_{q}^{u}(E)=$ $\hat{\mu}_{p}^{u}(F)$;

(2) $\hat{f}_{*} \hat{\mu}_{p}^{u}=\left.\lambda \hat{\mu}_{f(p)}^{u}\right|_{\hat{f}\left(\hat{W}_{\text {loc }}^{s}(p)\right)}$ where $\log \lambda$ is the entropy of $\left.f\right|_{\Lambda}$;

(3) $\operatorname{supp} \hat{\mu}_{p}^{u}=\hat{W}_{\text {loc }}^{s}(p)$.

The measures $\hat{\mu}_{p}^{u}$ are known as (unstable) transversal measures. Bowen and Marcus $[\mathrm{BM}]$ showed that transversal measures are unique up to rescaling by a factor independent of $p$. In fact, they are unique given only that they satisfy a more global variant of the first item in the conclusion of Theorem 1.2. We will not actually need this fact here, so we pursue it no further. We remark that the restriction is important on the right side of the equation in the second item of Theorem 1.2. Under our definition, there need not be an $\epsilon>0$ such that $\hat{W}_{\epsilon}^{s}(f(p)) \subset \hat{f}\left(\hat{W}_{\delta}^{s}(p)\right)$. Rather, if $\epsilon>0$ is small enough, we have

$$
\hat{W}_{\epsilon}^{s}(f(p)) \subset \bigcup_{p^{\prime} \in f^{-1}(f(p))} \hat{f}\left(\hat{W}_{\delta}^{s}\left(p^{\prime}\right)\right),
$$

where, since $\hat{f}$ is a homeomorphism, the sets in the union on the right side are mutually disjoint.

The proofs of existence and uniqueness for transversal measures were originally given for basic sets of Axiom A diffeomorphisms. These proofs rest principally on the existence and properties of so-called Markov partitions of a basic set. Careful examination of the literature $([\mathrm{A}],[\mathrm{B} 2],[\mathrm{KH}],[\mathrm{Sh}])$ reveals that one can establish all relevant results about Markov partitions (with proofs nearly unchanged) for any expansive homeomorphism $h: S \circlearrowleft$ of a compact metric space $S$ with the following shadowing property: given any $\epsilon>0$, there exists $\delta>0$ such that any for $\delta$ pseudoorbit $\left\{s_{j}\right\} \subset S$, there exists a unique point $s \in S$ such that $\operatorname{dist}\left(f^{j}(s), s_{j}\right)<$ $\delta$ for all $j \in \mathbf{Z}$. The shadowing property for $\hat{f}: \hat{\Lambda} \circlearrowleft$ holds as a consequence of local product structure (see [J1] for a proof). For those interested in further discussion of notions like hyperbolicity, shadowing, and local product structure for homeomorphisms, we recommend the last chapter of Akin's book [A].

Ruelle and Sullivan [RS] observed that transversal measures can be used to define a current $\sigma^{u}$ supported on the local unstable manifolds of $\Lambda$. For instance, if $\varphi$ is a test $(1,1)$ form supported on a small neighborhood of $p$, then the action of the laminar current $\sigma^{u}$ on $\varphi$ is given by

$$
\left\langle\sigma^{u}, \varphi\right\rangle=\int_{\hat{W}_{\mathrm{loc}}^{s}(p)}\left(\int_{W_{\mathrm{loc}}^{u}(\hat{q})} \varphi\right) d \hat{\mu}_{p}^{u}(\hat{q}) .
$$

Then $\sigma^{u}$ is extended to an entire neighborhood $\mathcal{N}$ of $\Lambda$ using a partition of unity. Holonomy invariance of transversal measures guarantees that the result is welldefined. Clearly, $\sigma^{u}$ is positive and $\operatorname{supp} \sigma^{u}=W_{\text {loc }}^{u}(\Lambda) \cap \mathcal{N}$. The property $f_{*} \sigma^{u}=$ $\lambda \cdot \sigma^{u}$ on $\mathcal{N}$ inherits from equation (1.1), the pushforward property of $\hat{\mu}_{p}^{u}$, and the fact that unstable manifolds are expanded by $f$. Finally, $\sigma^{u}$ is closed because any local unstable manifold $W_{\text {loc }}^{u}(\hat{q})$ will intersect small neighborhoods of $p$ in relatively compact subsets when $q$ is close to $p$. 
The preceding discussion can be modified to define holonomy along stable manifolds, stable transversal measures, and a laminar current supported on stable manifolds. Since we do not need these objects in this paper, we omit the details.

1.3. Saddle Sets in $\mathbf{P}^{2}$. Now we describe the situation of particular interest in this paper. We use $W_{\mathrm{loc}}^{u}(\Lambda)$ to denote the union of all local unstable manifolds associated with points $\hat{p} \in \hat{\Lambda}$.

Definition 1.3. Suppose $f: \mathbf{P}^{2} \circlearrowleft$ is holomorphic. We call $\Lambda$ a saddle set for $f$ if:

(1) $\Lambda$ is a hyperbolic set for $f$, and both $E^{s}$ and $E^{u}$ are one dimensional;

(2) $\left.f\right|_{\Lambda}$ is topologically transitive;

(3) $\hat{\Lambda}$ has local product structure;

(4) there exists a neighborhood $\mathcal{N}$ of $\Lambda$ such that $f^{n}\left(W_{\text {loc }}^{u}(\Lambda)\right) \cap \mathcal{N}=W_{\text {loc }}^{u}(\Lambda) \cap$ $\mathcal{N}$ for all $n \geq 0$.

Note that except for the last condition on $\Lambda$, the requirements of this definition are semi-local in nature - i.e. they only apply to the behavior of $f$ near $\Lambda$. Such sets $\Lambda$ arise naturally if we place a global restriction on the behavior of $f$. Recall that the non-wandering set $\Omega$ of $f$ consists of those points $p \in \mathbf{P}^{2}$ such that $f^{n}(U) \cap U \neq \emptyset$ for any neighborhood $U \ni p$ and arbitrarily large $n$. The map $f$ is Axiom $A$ if $f$ is hyperbolic on $\Omega$ and if in addition periodic points are dense in $\Omega$. Under these conditions, the set $\Omega$ decomposes into a finite number of closed sets, on each of which $f$ is topologically transitive. These are called the basic sets for $f$. By passing to a higher iterate and further decomposing, one can assume that $f$ is actually topologically mixing on each basic set.

If $\Lambda$ is a basic set for an Axiom A holomorphic map $f: \mathbf{P}^{2} \circlearrowleft$ with $\operatorname{dim} E^{s}(\Lambda)=$ $\operatorname{dim} E^{u}(\Lambda)=1$, then it turns out that $\Lambda$ is a saddle set. Condition 3. is proved in [J1, Proposition 3.3 on page 32]. A proof of 4. is given for diffeomorphisms in [BM, pp. 46-47].

Stable and unstable manifolds from different saddle sets $\Lambda, \Lambda^{\prime}$ of an Axiom A map can intersect in complicated ways. If $W_{\text {loc }}^{s}(\Lambda) \cap f^{n}\left(W_{\text {loc }}^{u}\left(\Lambda^{\prime}\right)\right) \neq \emptyset$ for some $n \geq 0$, then we say that $\Lambda \prec \Lambda^{\prime}$. When this relation actually orders the basic sets, then $f$ is said to satisfy the no cycles condition. We are interested in singling out basic sets $\Lambda$ of saddle type such that $\Lambda^{\prime} \nprec \Lambda$ for all other such $\Lambda^{\prime}$. These basic sets figured importantly in the study [FS2] (where they were referred to as "minimal") of hyperbolic holomorphic maps of $\mathbf{P}^{2}$ by Fornæss and Sibony. Since we do not wish to restrict ourselves to the Axiom A setting, we phrase our condition in terms of normal families.

Definition 1.4. Suppose that $\Lambda$ is a saddle set for a holomorphic map $f: \mathbf{P}^{2} \circlearrowleft$. We call $\Lambda$ terminal if for any $\hat{p} \in \hat{\Lambda}$ the iterates of $f$ restricted to $W_{\text {loc }}^{u}(\hat{p})-\Lambda$ form a normal family.

Our use of the word "terminal" is motivated by the case of Axiom A maps. If $f$ is Axiom $\mathrm{A}$ and an unstable manifold $W_{\text {loc }}^{u}(\hat{p})$ of $\Lambda$ does not intersect the stable manifolds of some other basic set, then $W_{\text {loc }}^{u}(\hat{p})-\Lambda$ must lie in the basins of attracting cycles. Hence basic sets of an Axiom A map which are "minimal" with respect to $\prec$ are necessarily terminal. On the other hand, iterates of $f$ cannot form a normal family in a neighborhood of any point in a stable manifold, because a disk transverse to the stable manifold will eventually be expanded. Therefore, if we 
assume that $W_{\text {loc }}^{u}(\Lambda)-\Lambda$ lies in the Fatou set of $f$, then $\Lambda$ must be minimal with respect to $\prec$. It would be interesting to know whether (or when) this apparently slightly stronger condition on $W_{\text {loc }}^{u}(\Lambda)$ is actually equivalent to terminality.

Before moving on, we mention that the "spectral decomposition" discussed above for basic sets of an Axiom A diffeomorphism applies to any saddle set, regardless of whether or not $f$ is Axiom A (see [KH], Theorem 18.3.1). In particular, we lose no generality by assuming that $f$ is topologically mixing on saddle sets.

1.4. Pluripotential Theory and Holomorphic Maps of $\mathbf{P}^{2}$. A fundamental tool for understanding complex dynamics on $\mathbf{P}^{k}$ is the use of pluripotential theory to construct and study positive closed currents with good transformation properties. The papers [HP], [U1], [FS1] present early applications of pluripotential theory to dynamics, and they remain excellent references.

A degree $d$ holomorphic map $f: \mathbf{P}^{2} \circlearrowleft$ acts linearly by pullback on the middle cohomology group. The group is freely generated by the cohomology class of the Fubini-Study Kähler form $\omega$ and $f^{*}$ multiplies this class by $d$. It is an interesting and very useful fact that there is a representative for the class of $\omega$ that is canonical for $f$. Namely, the sequence

$$
\frac{1}{d^{n}} f^{n *} \omega
$$

converges weakly to a positive closed $(1,1)$ current $T$ such that $f^{*} T=d \cdot T$. Positivity means that locally $T=d d^{c} u$ where $u$ is a plurisubharmonic function. In the particular case of $T$, the local potentials $u$ are always continuous. This allows us to consider the slice measure $\left.T\right|_{R}=d d^{c}(u \circ \iota)$ of $T$ along an embedded Riemann surface $\iota: R \rightarrow \mathbf{P}^{2}$. The following proposition concerning slice measures of $T$ is well-known.

Proposition 1.5. Slice measures of $T$ vary continuously. That is, if $\varphi$ is a test function in $\mathbf{P}^{2}$ and $R_{j} \subset \mathbf{P}^{2}$ are Riemann surfaces converging uniformly to a surface $R \subset \mathbf{P}^{2}$, then

$$
\left.\left.\int_{R_{j}} \varphi T\right|_{R_{j}} \rightarrow \int_{R} \varphi T\right|_{R}
$$

Slice measures transform according to the formula

$$
f_{*}\left(\left.T\right|_{R}\right)=\left.\frac{1}{d} \cdot T\right|_{f(R)}
$$

provided that $f$ is injective on $R$.

There is a remarkable characterization of $T$ in terms of normal families.

Theorem 1.6. The support of $T$ is equal to the complement of the Fatou set of $f-$ i.e. of the largest open set on which iterates of $f$ form a normal family. Likewise, if $R \subset \mathbf{P}^{2}$ is a Riemann surface, then $\left.\operatorname{supp} T\right|_{R}$ is the complement of the largest open subset of $R$ on which iterates of $f$ act normally.

A more recent paper [FS2] of Fornæss and Sibony gives another method of constructing positive closed $(1,1)$ currents from iterates of $f$. In order to state the next result, we recall that the mass $\mathrm{M}[S]$ of a current $S$ is given by

$$
\mathrm{M}[S]=\sup \left\{\langle S, \varphi\rangle:\|\varphi\|_{\infty} \leq 1\right\} .
$$


Theorem 1.7. Let $S$ be a positive closed $(1,1)$ current defined on an open set $U \subset \mathbf{P}^{2}$, and let $\psi: U \rightarrow \mathbf{C}$ be a smooth, compactly supported function. Then there is a constant $C$ such that $\mathrm{M}\left[f_{*}^{n}(\psi S)\right] \leq C d^{n}$ and $\mathrm{M}\left[\partial f_{*}^{n}(\psi S)\right] \leq C d^{n / 2}$ for all $n$. Therefore, the sequence $f_{*}^{n}(\psi S) / d^{n}$ has weak limit points and all such points are closed.

A construction of Bedford and Taylor [BT] allows us to understand the wedge product $S \wedge T$, where $S$ is a positive closed $(1,1)$ current on $U \subset \mathbf{P}^{2}$, as a positive measure. Locally, one chooses a continuous potential $u$ for $T$ and sets

$$
\int_{U} \psi S \wedge T=\left\langle S, u d d^{c} \psi\right\rangle
$$

It is shown in [FS2] that

Proposition 1.8. We have the transformation property

$$
f_{*}(S \wedge T)=\frac{f_{*} S}{d} \wedge T .
$$

In particular, if $S$ is defined on all of $\mathbf{P}^{2}$ and $f_{*} S=d \cdot S$, then $S \wedge T$ is an invariant measure.

\section{Proof of Theorem A}

Given a small neighborhood $\mathcal{N}$ of $\Lambda$, let $\sigma^{u}$ be the laminar current in $\mathcal{N}$ supported on local unstable manifolds. As indicated above, it is possible to interpret $\sigma^{u} \wedge T$ as a positive measure on $\mathcal{N}$. In this section we will study this measure carefully, ultimately proving Theorem A. Let us first show that the wedge product $\sigma^{u} \wedge T$ "commutes" with the laminar structure of $\sigma^{u}$.

Proposition 2.1. If $\varphi$ is a test function supported on a small neighborhood of $p \in \Lambda$, then

$$
\int_{U} \varphi \sigma^{u} \wedge T=\int_{\hat{W}_{\mathrm{loc}}^{s}(p)}\left(\left.\int_{W_{\mathrm{loc}}^{u}(\hat{q})} \varphi T\right|_{W_{\mathrm{loc}}^{u}(\hat{q})}\right) d \hat{\mu}_{p}^{u}(\hat{q}) .
$$

Proof. Note that by Proposition 1.5, the right side of the equation in the conclusion of this proposition defines a positive distribution (acting on $\varphi$ ) -i.e. it defines a Radon measure. Let $v$ be a local potential for $T$ on a neighborhood $U$ of the support of $\varphi$. Let $v_{j}$ be smooth plurisubharmonic functions that decrease uniformly locally to $v$ as $j$ goes to infinity. We can assume without loss of generality that $\varphi$ is smooth. Then

$$
\begin{aligned}
\int_{U} \varphi \sigma^{u} \wedge T & =\lim _{j \rightarrow \infty}\left\langle\sigma^{u}, v_{j} d d^{c} \varphi\right\rangle \\
& =\lim _{j \rightarrow \infty} \int_{\hat{W}_{\mathrm{loc}}^{s}(p)}\left(\int_{W_{\mathrm{loc}}^{u}(\hat{q})} v_{j} d d^{c} \varphi\right) d \hat{\mu}_{p}^{u}(\hat{q}) \\
& =\int_{\hat{W}_{\mathrm{loc}}^{s}(p)}\left(\int_{W_{\mathrm{loc}}^{u}(\hat{q})} v d d^{c} \varphi\right) d \hat{\mu}_{p}^{u}(\hat{q}) \\
& =\int_{\hat{W}_{\mathrm{loc}}^{s}(p)}\left(\left.\int_{W_{\mathrm{loc}}^{u}(\hat{q})} \varphi T\right|_{W_{\mathrm{loc}}^{u}(\hat{q})}\right) d \hat{\mu}_{p}^{u}(\hat{q})
\end{aligned}
$$


as desired. The first equality takes advantage of continuity of the wedge product operation under decreasing limits of plurisubharmonic functions (see [BT]). The third equality relies on the fact that, viewed as a function of $\hat{q}$, the inner integral on the second line converges uniformly to the inner integral on the third line. The last equality holds by definition.

Corollary 2.2. The support of $\sigma^{u} \wedge T$ contains $\Lambda$. The support of $\sigma^{u} \wedge T$ equals $\Lambda$ if and only if $\Lambda$ is terminal. In particular, if $\Lambda$ is terminal, then $\sigma^{u} \wedge T$ is independent of the choice of the neighborhood $\mathcal{N} \supset \Lambda$ on which $\sigma^{u}$ is defined.

Proof. Pick $p \in \Lambda$ and let $\hat{p}$ be a history. The support of $\left.T\right|_{W_{\text {loc }}^{u}(\hat{p})}$ contains $p$ because iterates of $f$ applied to $W_{\text {loc }}^{u}(\hat{p})$ do not form a normal family on any neighborhood of $p$. If $\varphi \geq 0$ is a continuous function equal to one at $p$, then

$$
\left.\int_{W_{\mathrm{loc}}^{u}(\hat{p})} \varphi T\right|_{W_{\mathrm{loc}}^{u}(\hat{p})}>0 .
$$

By continuity of slice measures the same is true for all $\hat{q} \in \hat{W}_{\text {loc }}^{s}(p)$ near $\hat{p}$. Therefore, Proposition 2.1 and the fact that supp $\hat{\mu}_{p}^{u}$ contains $\hat{p}$ imply that $\int \varphi \sigma^{u} \wedge T>0$. That is, $p \in \operatorname{supp} \sigma^{u} \wedge T$ and the first assertion is proved.

If $\Lambda$ is terminal then we have that $\left.T\right|_{W_{\text {loc }}^{u}(\hat{p})}$ is zero outside $\Lambda$, so another application of Proposition 2.1 shows that $\operatorname{supp} T \wedge \sigma^{u} \subset \Lambda$. If $\Lambda$ is not terminal, let $\hat{p} \in \hat{\Lambda}$ be a point such that $\left.\operatorname{supp} T\right|_{W_{\text {loc }}^{u}(\hat{p})}$ contains a point $q \notin \Lambda$. Continuous variation of slice measures and Proposition 2.1 again allow us to conclude that $q \in \operatorname{supp} \sigma^{u} \wedge T$.

Transversal measures are only determined up to constant multiples, so there is no canonical way to completely fix $\sigma^{u}$ in general. In the terminal case, however, we can normalize by rescaling $\sigma^{u}$ so that $\sigma^{u} \wedge T$ is a probability measure. The preceding results show that this defines the measure unambiguously.

We are now ready to prove the main result of this paper.

Proof of Theorem A. Let $\log \lambda$ be the entropy of $\left.f\right|_{\Lambda}$, and let $\sigma^{u}$ be the laminar current supported on local unstable manifolds. We can assume that $\sigma^{u}$ is defined on the neighborhood $\mathcal{N}$ of $\Lambda$ provided for in the definition of a saddle set. Suppose that $\chi$ is a cutoff function equal to one on some smaller neighborhood of $\Lambda$ but vanishing outside $\mathcal{N}$. Then by expansion along unstable manifolds and the transformation property of $\sigma^{u}$ we have a neighborhood $\mathcal{N}^{\prime} \subset \mathcal{N}$ of $\Lambda$ on which $\frac{1}{d^{n}} f_{*}^{n}\left(\chi \sigma^{u}\right) \geq \frac{\lambda^{n}}{d^{n}} \sigma^{u}$. Now Theorem 1.7 gives that pushforwards of $\chi \sigma^{u}$ have mass bounded by $C d^{n}$ for some $C$, so we conclude that $\lambda \leq d$.

If $\Lambda$ is terminal, then the results above show that $\nu:=\sigma^{u} \wedge T$ defines a probability measure on $\Lambda$. Now $\sigma^{u}$ has the invariance property $f_{*} \sigma^{u}=\lambda \cdot \sigma^{u}$ near $\mathcal{N}$, so by Proposition 1.8 we see that $f_{*} \nu=\frac{\lambda}{d} \cdot \nu$. But the mass of a positive measure is preserved under pushforward, so $\lambda=d$.

Finally suppose that $\lambda=d$ and that $\chi$ is as before. By Theorem 1.7, the sequence

$$
\sigma_{n}^{u}=\frac{1}{n} \sum_{j=1}^{n} \frac{1}{d^{j}} f_{*}^{j}\left(\chi \sigma^{u}\right) .
$$

has a subsequence that converges weakly to a positive closed $(1,1)$ current $\sigma_{\infty}^{u}$ on $\mathbf{P}^{2}$. Further, $f_{*} \sigma_{\infty}^{u}=d \cdot \sigma_{\infty}^{u}$. Since $f_{*} \sigma^{u}=d \cdot \sigma^{u}$ near $\Lambda$, we have $\sigma_{\infty}^{u} \geq \sigma^{u}$. The 
last condition in the definition of a saddle set gives in fact that $\sigma_{\infty}^{u}=\sigma^{u}$ near $\Lambda$. But the set $W_{\text {loc }}^{u}(\Lambda)$ is locally closed in $\mathcal{N}$, so we have that $\operatorname{supp} \sigma_{\infty}^{u} \cap \mathcal{N} \subset W_{\text {loc }}^{u}(\Lambda)$.

Because of the transformation property $f_{*} \sigma^{u}=d \cdot \sigma^{u}$, Proposition 1.8 gives us that the (finite) measure $\nu=\sigma_{\infty}^{u} \wedge T$ is $f$-invariant. In particular, the Poincaré recurrence theorem tells us that $\nu$ almost every point is recurrent. But $\operatorname{supp} \nu \cap \mathcal{N}^{\prime} \subset$ $W_{\text {loc }}^{u}(\Lambda)$ for the neighborhood $\mathcal{N}^{\prime} \supset \Lambda$ described above, and the last condition in the definition of a saddle set guarantees that the only recurrent points in $W_{\text {loc }}^{u}(\Lambda)$ are those in $\Lambda$. We conclude that $\operatorname{supp} \nu \cap \mathcal{N} \subset \Lambda$. Near $\Lambda$ we have that $\nu=$ $\sigma_{\infty}^{u} \wedge T=\sigma^{u} \wedge T$, so it follows from Corollary 2.2 that $\Lambda$ is terminal.

We remark that a more general version of Theorem A can be had with essentially the same proof we have just given. If $f: \mathbf{P}^{k} \circlearrowleft$ is a holomorphic map and $\Lambda \subset \mathbf{P}^{k}$ is a saddle set with a single expanding direction, then one can again show that $h_{\text {top }}\left(\left.f\right|_{\Lambda}\right) \leq \log d$ and that equality holds if and only if $\Lambda$ is terminal. The definitions of saddle set and terminal are, moreover, exactly the same as the ones we have used here.

Our proof does not work as is, however, for saddle sets with $l>1$ expanding directions. The problem here is that the appropriate slice measures to consider are of the form $\left.T^{l}\right|_{W_{\text {loc }}^{u}(\hat{p})}, \hat{p} \in \hat{\Lambda}$. Whereas the support of a slice of $T$ is equal to the set on which iterates of $f$ fail to be normal, no such relationship is known for slices of $T^{l}$. In particular, there is no guarantee that slices of $T^{l}$ are nonzero on any of the local unstable manifolds.

\section{Examples-Proof of Theorem B}

In this section we will give several examples of saddle sets in $\mathbf{P}^{2}$, both terminal and nonterminal. The latter ones are significantly harder to construct and their existence constitutes the statement of Theorem B.

3.1. Terminal saddle sets. Terminal saddle sets in $\mathbf{P}^{2}$ are easy to find.

Example 3.1. Let $p$ and $q$ be polynomial mappings of $\mathbf{C}$ of common degree $d \geq 2$, and let $f(z, w)=(p(z), q(w))$ be the product map. Assume that $q$ is hyperbolic and let $A_{q}$ be the set of attracting periodic points of $q$. If $z$ is an attracting fixed point of $p$ and $J_{q}$ is the Julia set of $q$, then $\Lambda:=\{z\} \times J_{q}$ is a terminal, mixing saddle set for $f$. Indeed, if $\hat{r} \in \hat{\Lambda}$, then $W_{\text {loc }}^{u}(\hat{r})-\Lambda=\{z\} \times\left(\mathbf{C}-J_{q}\right)$, so if $U \subset \subset W_{\text {loc }}^{u}(\hat{r})-\Lambda$ is connected, then $f^{n}$ converges uniformly on $U$ to some point in $\left(\{z\} \times A_{q}\right) \cup\{[0: 1: 0]\}$. Thus $\Lambda$ is terminal.

The next two examples are quite similar to the first one.

Example 3.2. This example uses a construction of Ueda [U2]. Namely, let $g$ be a rational map of $\hat{\mathbf{C}} \simeq \mathbf{P}^{1}$ of degree $d \geq 2$. There exists a branched covering $\pi: \mathbf{P}^{1} \times \mathbf{P}^{1} \rightarrow \mathbf{P}^{2}$, which semiconjugates $g \times g$ to a holomorphic mapping $f: \mathbf{P}^{2} \circlearrowleft$ of degree $d$. If $g$ is hyperbolic with Julia set $J_{g}$ and $z$ is an attracting fixed point for $g$, then $\Lambda:=\pi\left(\{z\} \times J_{g}\right)$ is a terminal, mixing saddle set for $f$.

Example 3.3. Let $f(z, w)=(p(z, w), q(z, w))$ be a polynomial mapping of $\mathbf{C}^{2}$ of degree $d \geq 2$. Assume that $f$ is regular, i.e. that $f$ extends to a holomorphic mapping of $\mathbf{P}^{2}$ (see [BJ]). The line at infinity $\Pi:=\mathbf{P}^{2}-\mathbf{C}^{2} \simeq \mathbf{P}^{1}$ is completely invariant and the restriction $f_{\Pi}$ of $f$ to $\Pi$ is a rational map $f_{\Pi}$, the Julia set of which we denote by $J_{\Pi}$. If $f_{\Pi}$ is hyperbolic, then $\Lambda:=J_{\Pi}$ is a terminal, mixing saddle set for $f$ in $\mathbf{P}^{2}$. 
Remark 3.4. In the above three examples the unstable currents $\sigma^{u}$ are given as currents of integration on the curves $\{z\} \times \mathbf{C}, \pi\left(\{z\} \times \mathbf{P}^{1}\right)$ and $\Pi$, respectively. Perturbations of these examples will yield new terminal saddle sets. Indeed, if $\Lambda$ is a terminal saddle set for a holomorphic mapping $f: \mathbf{P}^{2} \circlearrowleft$ such that $W_{\text {loc }}^{u}(\Lambda)-\Lambda$ is in the union of basins of attraction of finitely many sinks, then any small perturbation of $f$ will have a terminal saddle set close to $\Lambda$. For perturbations of the three examples, the current $\sigma^{u}$ will, in general, not be a current of integration on an analytic set.

Example 3.5. Consider a polynomial automorphism of $\mathbf{C}^{2}$ of the form $f_{0}(z, w)=$ $(p(z)+a w, z)$, where $p$ is a polynomial of degree $d \geq 2$. Assume that $f_{0}$ is hyperbolic, i.e. that $J$ is a hyperbolic set (see [BS]). Let $f(z, w)=\left(p(z)+a w, z+\epsilon w^{d}\right)$ for small $\epsilon>0$. Then $f$ has a terminal saddle set $\Lambda$, which is a perturbation of $J$.

3.2. Nonterminal saddle sets. We now prove Theorem B by constructing mappings with nonterminal saddle sets in $\mathbf{P}^{2}$. The examples that we will describe belong to the family of polynomial skew products on $\mathbf{C}^{2}$ - a class of nontrivial holomorphic mappings on $\mathbf{P}^{2}$ with tractable dynamics.

We start by recalling some facts about skew products on $\mathbf{C}^{2}$. Then we state sufficient conditions for a skew product to be Axiom A and have a nonterminal saddle set. Finally, we show how to construct explicit examples where these conditions are satisfied.

3.2.1. Polynomial skew products on $\mathbf{C}^{2}$. These are mappings of $\mathbf{C}^{2}$ of the form

$$
f(z, w)=(p(z), q(z, w))
$$

where $p$ and $q$ are polynomials of the same degree $d \geq 2$, and $q$ has nonvanishing $w^{d}$-term. Polynomial skew products have been studied by Heinemann [H1, H2], by Sester [Se1, Se2], and by one of the authors [J2, J3]. We recall a few definitions and results from [J2].

The first component of (3.1) defines a polynomial mapping of $\mathbf{C}$, the Julia set and filled Julia set of which are denoted by $J_{p}$ and $K_{p}$, respectively. The polynomial $p$ is said to be uniformly expanding on $J_{p}$ (or hyperbolic) if there exist $c>0$ and $\lambda>1$ such that $\left|D p^{n}(z)\right|>c \lambda^{n}$ for $z \in J_{p}$ and $n \geq 1$.

We write $q_{z}$ for the polynomial mapping $q(z, \cdot)$ of $\mathbf{C}$, defined by (3.1) and we denote the composition $q_{z_{n-1}} \circ \cdots \circ q_{z}$ by $Q_{z}^{n}$. If $z \in K_{p}$, then we denote by $K_{z}$ the filled Julia set of $\left\{Q_{z}^{n}\right\}_{n \geq 1}$, i.e. the set where this family is bounded. Also, we set $J_{z}=\partial K_{z}$. If $Z \subset K_{p}$ is compact and $p(Z) \subset Z$, then we say that $f$ is vertically expanding over $Z$ if there exists $c>0$ and $\lambda>1$ such that $\left|D Q_{z}^{n}(w)\right| \geq c \lambda^{n}$ for $z \in Z, w \in J_{z}$ and $n \geq 1$.

A polynomial skew product (3.1) of $\mathbf{C}^{2}$ extends to a holomorphic mapping of $\mathbf{P}^{2}$. The line at infinity $\Pi:=\mathbf{P}^{2}-\mathbf{C}^{2} \simeq \mathbf{P}^{1}$ is completely invariant and the restriction $f_{\Pi}$ of $f$ to $\Pi$ is a polynomial map $f_{\Pi}$, the Julia set of which we denote by $J_{\Pi}$.

The following result characterizes Axiom A for polynomial skew products:

Theorem 3.6. [J2, Theorem 8.2] A polynomial skew product (3.1), viewed as a holomorphic mapping of $\mathbf{P}^{2}$, is Axiom $A$ if and only if:

(i) $p$ is uniformly expanding on $J_{p}$;

(ii) $f$ is vertically expanding over $J_{p}$;

(iii) $f$ is vertically expanding over $A_{p}$, the set of attracting periodic points for $p$; 
(iv) $f_{\Pi}$ is uniformly expanding on $J_{\Pi}$.

The conditions (i)-(iv) are most easily checked in terms of the postcritical set. Indeed, it is a standard one-dimensional result that a rational map is uniformly expanding on its Julia set if and only if its postcritical set is disjoint from the Julia set. A generalization of this holds for skew products. Let $C_{z}$ denote the critical set of $q_{z}$ for $z \in \mathbf{C}$. Let $Z \subset K_{p}$ be compact and invariant, and let $D_{Z}$ denote the postcritical set over $Z$, i.e.

$$
D_{Z}=\overline{\bigcup_{n \geq 1} f^{n}\left(\bigcup_{z \in Z}\{z\} \times C_{z}\right)}=: \bigcup_{z \in Z}\{z\} \times D_{Z, z},
$$

where the last equation defines $D_{Z, z}$. Also, let

$$
J_{Z, z}^{*}=\limsup _{\zeta \rightarrow z, \zeta \in Z} J_{z}
$$

in the Hausdorff metric ( $z \rightarrow J_{z}$ is not continuous in general).

Theorem 3.7. [J2, Theorem 3.1] $f$ is vertically expanding over $Z$ if and only if $D_{Z, z} \cap J_{Z, z}^{*}=\emptyset$ for all $z \in Z$.

The most important situation is when $Z=J_{p}$. We then write $D_{z}:=D_{J_{p}, z}$ and $J_{z}^{*}:=J_{J_{p}, z}^{*}$.

3.2.2. A sufficient condition. The following proposition gives a sufficient condition for a polynomial skew product on $\mathbf{C}^{2}$ to be Axiom $\mathrm{A}$ and to have a nonterminal saddle set with topological entropy $\log k$ for some integer $k$ less than the degree of the skew product. In Section 3.2.3 we will show how to construct skew products satisfying the conditions in the proposition below. Taken together, this will provide a proof of Theorem B.

Proposition 3.8. Let $1 \leq k<d$ and let $f(z, w)=(p(z), q(z, w))$ be a polynomial skew product of degree $d$ on $\mathbf{C}^{2}$ such that $q_{z}(w)=w^{d}+r(z)$ for some polynomial $r$ of degree $<d$ and such that:

(i) there exist $\lambda>1$, a compact set $D \subset \mathbf{C}$ and d disjoint subsets $D_{j}$ of $D$ such that

$$
p^{-1}(D)=D_{1} \cup \cdots \cup D_{d}
$$

and $p: D_{j} \rightarrow D$ is a homeomorphism with $|D p(z)| \geq \lambda, z \in D_{j}, 1 \leq j \leq d$;

(ii) there exist numbers $M \geq 3, A \geq M+2$ such that if we write

$$
E=D_{1} \cup \cdots \cup D_{k} \quad \text { and } \quad E^{\prime}=D_{k+1} \cup \cdots \cup D_{d} \text {, }
$$

then
(a) $|r(z)| \leq 1 / 5$ for $z \in E$;
(b) $3 A \leq|r(z)| \leq M A$ for $z \in E^{\prime}$.

Then $f$ is Axiom $A$ on $\mathbf{P}^{2}$ and has a nonterminal basic set $\Lambda$ with $h_{\mathrm{top}}\left(\left.f\right|_{\Lambda}\right)=\log k$.

The idea is that if $z \in E$, then $q_{z}(w) \approx w^{d}$, whereas if $z \in E^{\prime}$, then $q_{z}(w)=$ $w^{d}+r(z)$, where is $r(z)$ is large. The set $\Lambda$ will be the closure of the set of all periodic saddle points $(z, w)$ with $z \in E$. 
Proof of Proposition 3.8. It follows easily from (i) that $J_{p}=\bigcap_{n \geq 0} p^{-n}(D)$ and that $p$ is uniformly expanding on $J_{p}$. In fact $\left.p\right|_{J_{p}}$ is isomorphic to the full shift on $d$ symbols. Let $L$ be the set of $z \in J_{p}$ such that $z_{n} \in E$ for all $n \geq 0$. Then $L$ is compact and $\left.p\right|_{L}$ is isomorphic to the full shift on $k$ symbols.

We will estimate the position of the Julia set $J_{z}$ and the postcritical set $D_{z}$ for $z \in J_{p}$. Using the triangle inequality we easily show

$$
\begin{array}{rll}
z \in E \cup E^{\prime},|w| \geq A & \Longrightarrow & \left|q_{z}(w)\right| \geq 2|w| \\
z \in E^{\prime},|w| \leq 1 / 3 & \Longrightarrow & \left|q_{z}(w)\right| \geq 2 A \\
z \in E,|w| \leq 1 / 3 & \Longrightarrow & \left|q_{z}(w)\right| \leq 14 / 45 .
\end{array}
$$

It follows from this that

$$
\begin{array}{rll}
z \in J_{p} & \Longrightarrow & K_{z} \subset \mathbf{D}_{A} \\
z \in L & \Longrightarrow & \mathbf{D}_{1 / 3} \subset K_{z} \\
z \in J_{p}-L & \Longrightarrow & \mathbf{D}_{1 / 3} \cap K_{z}=\emptyset .
\end{array}
$$

Since $C_{z}=\{0\}$ for all $z \in \mathbf{C}$ we also get

$$
z \in J_{p} \quad \Longrightarrow \quad D_{z} \subset \mathbf{D}_{14 / 45} \cup\left(\mathbf{C}-\mathbf{D}_{2 A}\right) \text {. }
$$

We are now in position to prove that $f$ is Axiom A, using Theorem 3.6. Indeed, $p$ is uniformly expanding on $J_{p}$ by (i) and $f_{\Pi}$ is uniformly expanding on $J_{\Pi}$, since $f_{\Pi}$ is affinely conjugate to $\zeta \rightarrow \zeta^{d}$. Further, (3.6)-(3.8) imply that $d\left(D_{z}, J_{z}^{*}\right) \geq 1 / 45$ for all $z \in J_{p}$, so by Theorem $3.7 f$ is vertically expanding over $J_{p}$. Since $p$ has no attracting periodic points, $f$ must be Axiom $\mathrm{A}$ on $\mathbf{P}^{2}$ by Theorem 3.6.

From the proof of Theorem 3.6 it follows that the nonwandering set of $f$ can be written as $\Omega=A_{\Pi} \cup J_{\Pi} \cup J_{2} \cup \Lambda$. Here $A_{\Pi}$ is the set of sinks of $f_{\Pi}$, and $J_{\Pi}$ is the Julia set of $f_{\Pi}$. Further, $J_{2}=\bigcup_{z \in J_{p}}\{z\} \times J_{z}$ is the closure of the repelling periodic points of $f$ and $\Lambda$ is the closure of the periodic saddle points $(z, w)$ with $z \in J_{p}$.

We analyze the set $\Lambda$ in more detail. If $(z, w)$ is a periodic point in $\Lambda$, say of period $n$, then $p^{n}(z)=z$ and $w \in \operatorname{int} K_{z}$. Suppose that $z \notin L$. Then it follows from (3.2)-(3.4) that all critical points of $Q_{z}^{n}$ have unbounded orbits. Thus $\operatorname{int} K_{z}=\emptyset$, a contradiction. We conclude that all periodic points $(z, w) \in \Lambda$ have $z \in L$.

By (3.4) we have $q_{z}\left(\mathbf{D}_{1 / 3}\right) \subset \mathbf{D}_{14 / 45}$ for $z \in L$. A theorem of Sester [Se2] applied to the restriction of $f$ to $L \times \mathbf{C}$ then shows that $K_{z}$ is a quasidisk for all $z \in L$.

Let $(\hat{L}, \hat{p})$ be the natural extension of $\left.p\right|_{L}$. If $\hat{z}=\left(z_{i}\right)_{i \leq 0}$ is a history in $\hat{L}$ then we define $\theta(\hat{z}) \in \Lambda$ by

$$
\theta(\hat{z})=\bigcap_{k \geq 0} q_{z_{-k}}^{k}\left(\mathbf{D}_{1 / 3}\right)
$$

This is well defined by (3.4) and $\theta$ is continuous on $\hat{L}$. Write

$$
\tilde{\Lambda}:=\bigcup_{\hat{z} \in \hat{L}} \theta(\hat{z}) \text {. }
$$

Then $\tilde{\Lambda}$ is compact. We claim that $\tilde{\Lambda}=\Lambda$. To see this, first notice that if $\hat{z}$ is a periodic history, say of order $n$, then $\theta(\hat{z})$ coincides with the (unique) fixed point of $Q_{z}^{n}$. Thus every periodic saddle point in $L \times \mathbf{C}$ is contained in $\tilde{\Lambda}$ so $\Lambda \subset \tilde{\Lambda}$.

To prove the reverse inclusion we show that $f$ is topologically mixing on $\tilde{\Lambda}$. The argument is adapted from [JW]. Let $U_{1}$ and $U_{2}$ be two open sets in $\mathbf{C}^{2}$ with 
$U_{i} \cap \tilde{\Lambda} \neq \emptyset$ for $i=1,2$. We will show that $f^{n}\left(U_{1}\right) \cap U_{2} \neq \emptyset$ for large $n$. Pick $x \in U_{2} \cap \tilde{\Lambda}$ and write $x=\theta(\hat{z})$ for some $\hat{z} \in \hat{L}$. We can find $k \geq 0$ such that $f^{k}\left(\left\{z_{-k}\right\} \times V_{z_{-k}}\right) \subset \subset U_{2}$. By continuity there is an open subset $\omega$ of $z_{-k}$ in $L$ such that $f^{k}\left(\{z\} \times V_{z_{-k}}\right) \subset \subset U_{2}$ for $z \in \omega$. Let $\omega_{1}$ be the projection of $U_{1}$ on the $z$-axis. Since $p$ is topologically mixing on $L$ there exists $N \geq 0$ such that if $n \geq N$, then $p^{n}\left(\omega_{1}\right) \cap \omega \neq \emptyset$. It follows that $f^{n}\left(U_{1}\right) \cap U_{2}$ for $n \geq k+N$. Thus $f$ is topologically mixing on $\tilde{\Lambda}$ and $\tilde{\Lambda}=\Lambda$.

Finally, we claim that the topological entropy of $\left.f\right|_{\Lambda}$ equals $\log k$. Since $d<k$, Theorem A then shows that $\Lambda$ is nonterminal. On the one hand, the projection $(z, w) \rightarrow z$ semiconjugates $\left.f\right|_{\Lambda}$ to $\left.p\right|_{L}$, so

$$
h_{\text {top }}\left(\left.f\right|_{\Lambda}\right) \geq h_{\text {top }}\left(\left.p\right|_{L}\right)=\log k,
$$

since $\left.p\right|_{L}$ is isomorphic to the full shift on $k$ symbols. On the other hand, by a result of Bowen [B1] we have

$$
h_{\text {top }}\left(\left.f\right|_{\Lambda}\right) \leq h_{\text {top }}\left(\left.p\right|_{L}\right)+\sup _{z \in L} h_{\text {top }}(f, \Lambda \cap\{z\} \times \mathbf{C})=\log k,
$$

since $h_{\text {top }}(f, \Lambda \cap\{z\} \times \mathbf{C})=0$ for $z \in L$ by contraction. This completes the proof that $h_{\text {top }}\left(\left.f\right|_{\Lambda}\right)=\log k$.

3.2.3. Satisfying the hypotheses. We now show that we can find, for every $d$ and $k$, a polynomial skew product on $\mathbf{C}^{2}$ that satisfies the assumptions of Proposition 3.8. The following example, which is similar to [J2, Example 9.6], does the job for $d=2$, $k=1$.

Example 3.9. Let $f(z, w)=\left(z^{2}-9900, w^{2}+(99+z) / 6\right)$. We take $D=[-100,100]$, $D_{1}=[-100,-b], D_{2}=[b, 100]$ where $b=\sqrt{9800} \approx 98.99$. Then $|D p(z)|=2|z| \geq$ 196 for $z \in D_{1} \cup D_{2}$. Further, $r(z)=(99+z) / 6$ so we have $|r(z)| \leq 1 / 5$ when $z \in D_{1}$ and $32 \leq|r(z)| \leq 34$ when $z \in D_{2}$. Thus we can take $A=10, M=4$. In this case the nonterminal basic set $\Lambda$ is the single saddle fixed point $(-99,0)$.

We now turn to the general case with $1 \leq d<k$.

Let $p_{0}(z)$ be a polynomial of degree $d$ with exactly $d$ different zeros $\xi_{1} \ldots \xi_{d}$. Let $a>0$ be a large number and define $p(z)=a \cdot p_{0}(z)$. Fix a closed disk $D$ centered at the origin and containing all the points $\xi_{j}$ in its interior. If $a$ is large enough, then $p^{-1}(D)$ is contained in the interior of $D$ and consists of $d$ disjoint Jordan domains $D_{j}, j=1 \ldots, d$. Further, the restriction of $p$ maps $D_{j}$ homeomorphically onto $D$ and $|D p| \geq 2$ on $D_{j}$. Notice that $\operatorname{diam}\left(D_{j}\right) \sim 1 / a$.

Pick an integer $k$ with $1 \leq k<d$, let $E$ be the union of $k$ of the sets $D_{j}$ and let $E^{\prime}$ be the union of the remaining sets $D_{j}$. Define

$$
r_{0}(z)=\prod_{\xi_{j} \in E}\left(z-\xi_{j}\right)
$$

Then there exist constants $m_{0}, m_{1}$ and $m_{2}$ such that

$$
\left|r_{0}(z)\right| \leq m_{2} / a \text { for } z \in E \text { and } m_{0} \leq\left|r_{0}(z)\right| \leq m_{1} \text { for } z \in E^{\prime}
$$

for all large enough $a$. Now let $r(z)=\frac{a}{5 m_{2}} r_{0}(z), A=\frac{a m_{0}}{15 m_{2}}, M=\frac{3 m_{1}}{m_{0}}$. Then, for large $a$, we have $M \geq 3, A \geq M+2$,

$$
|r(z)| \leq 1 / 5 \text { for } z \in E \text { and } 3 A \leq|r(z)| \leq M A \text { for } z \in E^{\prime} .
$$

This completes the construction and hence the proof of Theorem B. 


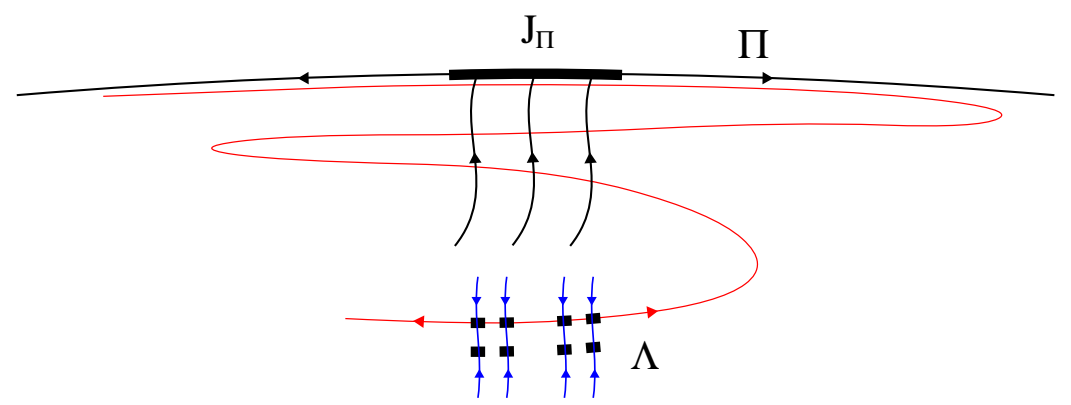

Figure 1. A nonterminal basic set. The picture shows how forward images of a local unstable manifold of a history in $\Lambda$ intersect local stable manifolds of points in $J_{\Pi}$, and hence accumulate on all of $\Pi$.

Remark 3.10. It is possible to say more precisely how the set $\Lambda$ in Proposition 3.8 fails to be terminal. Namely, if $\hat{r} \in \hat{\Lambda}$, then $f^{n}\left(W_{\text {loc }}^{u}(\hat{r})\right)$ will have transverse intersections with local stable manifolds of points in $J_{\Pi}$ for large $n$. This implies that $f^{n}\left(W_{\text {loc }}^{u}(\hat{p})\right)$ will accumulate on all of $\Pi$ (see Figure 1$)$. In particular, $\left\{f^{n}\right\}$ fails to be normal on $W_{\text {loc }}^{u}(\hat{r})-\Lambda$.

\section{REFERENCES}

[A] E. Akin. The general topology of dynamical systems. American Mathematical Society, Providence, RI, 1993.

[BJ] E. Bedford and M. Jonsson. Regular polynomial endomorphisms of $\mathbf{C}^{k}$. Stony Brook IMS Preprint \#1998/3.

[BS] E. Bedford and J. Smillie. Polynomial diffeomorphisms of $\mathbf{C}^{2}$ : Currents, equilibrium measure and hyperbolicity. Invent. Math. 103 (1991), 69-99.

[BT] E. Bedford and B. A. Taylor. The Dirichlet problem for a complex Monge-Ampère equation. Invent. Math. 37 (1976), 1-44.

[B1] R. Bowen. Entropy for group endomorphisms and homogeneous spaces. Trans. Amer. Math. Soc. 153 (1971), 401-414.

[B2] R. Bowen. Equilibrium states and the ergodic theory of Anosov diffeomorphisms. SpringerVerlag, Berlin-New York, 1975. Lecture Notes in Mathematics, Vol. 470.

[BM] R. Bowen and B. Marcus. Unique ergodicity for horocycle foliations. Isr. J. Math. 26 (1977), $43-67$.

[FS1] J. E. Fornæss and N. Sibony. Complex dynamics in higher dimension II. In Modern Methods in Complex Analysis (T. Bloom et al., editors), number 137 in Annals of Mathematics Studies, pages 135-182. Princeton University Press, 1995.

[FS2] J. E. Fornæss and N. Sibony. Hyperbolic maps on $\mathbf{P}^{2}$. Math. Ann. 311 (1998), 305-333.

[H1] S.-M. Heinemann. Julia sets for holomorphic endomorphisms of $\mathbf{C}^{n}$. Ergodic Theory Dynam. Systems 16 (1996), 1275-1296.

[H2] S.-M. Heinemann. Julia sets of skew products in $\mathbf{C}^{2}$. Kyushu J. Math. 52 (1998), 299-329.

[HP] J. H. Hubbard and P. Papadopol. Superattractive fixed points in $\mathbf{C}^{n}$. Indiana Univ. Math. J. 43 (1994), 321-365.

[J1] M. Jonsson. Dynamical Studies in Several Complex Variables. PhD thesis, Royal Institute of Technology, November 1997.

[J2] M. Jonsson. Dynamics of polynomial skew products on $\mathbf{C}^{2}$. To appear in Math. Ann.

[J3] M. Jonsson. Ergodic properties of fibered rational maps and applications. Preprint.

[JW] M. Jonsson and B. Weickert. A nonalgebraic attractor in $\mathbf{P}^{2}$. Accepted by Proc. Amer. Math. Soc. 
[KH] A. Katok and B. Hasselblatt. Introduction to the modern theory of dynamical systems. Cambridge University Press, Cambridge, 1995.

[PS] C. Pugh and M. Shub. Ergodic attractors. Trans. Amer. Math. Soc. 312 (1989), 1-54.

[RS] D. Ruelle and D. Sullivan. Currents, flows and diffeomorphisms. Topology 14 (1975), 319327.

[Se1] O. Sester. Étude dynamique des polynômes fibrés. PhD thesis, Université de Paris-Sud, 1997.

[Se2] O. Sester. Hyperbolicité des polynômes fibrés. Preprint.

[Sh] M. Shub. Global stability of dynamical systems. Springer-Verlag, 1987.

[U1] T. Ueda. Fatou sets in complex dynamics on projective spaces. J. Math. Soc. Japan 46 (1994), 545-555.

[U2] T. Ueda. Complex dynamical systems on projective spaces. Preprint.

Department of Mathematics, University of Notre Dame, Notre Dame, IN 46556

E-mail address: jdiller@nd.edu

Department of Mathematics, University of Michigan, Ann Arbor, Mi 48109-1109

E-mail address: mattiasj@umich.edu 